ISSN 0001-6012/2015/57/4/195-196 Acta Médica Costarricense, ( 2015 Colegio de Médicos y Cirujanos de Costa Rica

\title{
Opinión
}

\section{Salud mental y derechos humanos: el caso de los seguros médicos privados}

\section{(Mental health and human rights: The case of private health insurance)}

Ricardo Millán-González y Olger Ignacio González-Espinoza²

\section{Resumen}

La salud es un derecho fundamental reconocido por la Constitución Política de Costa Rica y diversos tratados internacionales, de los cuales el Estado es parte. A pesar de lo anterior, muchos contratos de seguro médico privado excluyen (o restringen severamente) diversas condiciones médicas, entre las que se encuentran las clasificadas como psiquiátricas. Este tipo de prácticas resulta no solo estigmatizante, sino que también revela una falta de garantía de la protección integral de la salud general de las personas aseguradas. Resulta indispensable, por lo tanto, que las aseguradoras modifiquen su postura de continuar excluyendo servicios básicos, mientras que el Estado, por su parte, debe ejercer su obligación de velar por la efectiva garantía de los derechos humanos, en particular mediante una adecuada supervisión del derecho a la salud.

Descriptores: seguros médicos privados, derechos humanos, salud mental, derecho a la salud

\section{Abstract}

Health is recognized as a fundamental right by the Political Constitution of Costa Rica and by several international treaties ratified by the State. In spite of this, many private insurance companies exclude (or heavily restrict) coverage of several medical conditions, among them, those classified as psychiatric. This type of practice is not only discriminatory, but also reveals a failure to guarantee an integral protection of people's health. It is therefore essential that insurance companies abstain from excluding basic health services, while the State, in turn, must comply with its obligation to ensure the effective guarantee of human rights, in particular by supervising the adequate respect of the right to health.

Keywords: private medical insurance, human rights, mental health, right to health.

Fecha recibido: 16 de marzo 2015

Fecha aprobado: 18 de junio 2015

En Costa Rica, las empresas de seguros de servicios de salud explícitamente excluyen de sus contratos de adhesión, o en el mejor de los casos restringen de forma muy significativa, la cobertura de determinados padecimientos, entre los cuales se encuentran las enfermedades

Afiliación de los autores: 'Psiquiatría de Enlace, UCR, Hospital Clínica Bíblica, ${ }^{2}$ Secretaría de la Corte Interamericana de Derechos Humanos.

凶ricardo.millangonzalez@ucr.ac.cr mentales. Es decir, en caso de que una persona sufra alguna de estas afecciones, se ve obligada a pagar por su cuenta el tratamiento médico y farmacológico que requiera, o a no recibirlo del todo si ello excede sus posibilidades económicas, a pesar de lo indispensable que pueda ser en un momento determinado. En ciertas ocasiones, podría recibir un monto de cobertura muy limitado, si se compara con otras enfermedades.

Los trastornos afectivos, ansiosos, psicóticos, por síntomas somáticos y demencias, entre otros, son entidades médicas bien definidas y reconocidas por la Organización Mundial de la Salud (OMS) y la Asociación Americana de Psiquiatría, y tipificadas y caracterizadas por ellas 
mismas, en la Clasificación Internacional de Enfermedades en su décima versión, ${ }^{1}$ y en el Manual Diagnóstico y Estadístico de los Trastornos Mentales en su quinta versión. ${ }^{2}$ Se trata de condiciones clínicas reconocidas, altamente prevalentes, diagnosticables y tratables, y capaces de contribuir a la carga total de enfermedad, según se ha evidenciado cuando se expresa a través de medidas como los disability-adjusted life-year (DALYs), que es la suma de años vividos con discapacidades y años perdidos. ${ }^{3}$ En este sentido, el Movimiento de Salud Mental Global ha expuesto que las condiciones psiquiátricas y neuropsiquiátricas hacen no solo una contribución directa, sino también indirecta en la carga total de enfermedad, a través del incremento del riesgo de enfermedades comunicables y no comunicables, de sus consecuencias, y de la capacidad de que estas sean detectadas y correctamente tratadas. ${ }^{4}$

La salud es un derecho humano. Así está reconocido en la Constitución Política del Estado de Costa Rica ${ }^{5}$ y en los tratados internacionales ratificados. En el primer caso, se cita textualmente en el artículo 46: “(...) Los consumidores y usuarios tienen derecho a la protección de su salud, ambiente, seguridad e intereses económicos; a recibir información adecuada y veraz; a la libertad de elección, y a un trato equitativo (...)". Por su parte, el Protocolo a la Convención Americana sobre Derechos Humanos relativo a los Derechos Económicos, Sociales y Culturales o Protocolo de San Salvador, ${ }^{6}$ define el derecho a la salud como "el disfrute del más alto nivel de bienestar físico, mental y social" y establece que, con el fin de hacerlo efectivo, "los Estados Partes se comprometan a reconocer la salud como un bien público" y, entre otras medidas, a garantizarlo mediante "la extensión de los beneficios de los servicios de salud a todos los individuos sujetos a la jurisdicción del Estado". De hecho, en informes de la OMS se ha contemplado la posibilidad de exigir a las aseguradoras públicas y privadas, que incluyan determinados servicios de salud mental en el paquete básico ofrecido a todos sus clientes.

Es claro que, en el caso de muchos pacientes y usuarios de servicios privados, se presenta una restricción a su derecho a la salud mental, la cual, en principio, se podría pensar que está consentida por haber sido así aceptada al firmar el contrato de seguro. Sin embargo, más allá de que las empresas no admiten modificaciones en los contratos que ofrecen, existe una discriminación implícita en tal exclusión, pues la atención en salud se niega en forma estigmatizante y arbitraria a quien la requiere ante una condición particular imprevisible y, por ende, se restringe en los mismos términos, sus derechos sanitarios. El problema es, además, que esa restricción es avalada indirectamente por el Estado, el cual ha autorizado la oferta de los seguros privados de salud y, en este sentido, no los ha regulado o fiscalizado adecuada y efectivamente.

La exclusión de tales padecimientos de los contratos de seguros, o su restricción excesiva a coberturas insuficientes, constituyen una forma de cláusula abusiva, por estar sustentada en una sutil pero flagrante forma de discriminación. En el fondo, lo anterior se explica por una realidad: los tratamientos psicológicos o psiquiátricos pueden ser muy costosos, sobre todo por la cantidad y lo prolongado de las terapias y el alto precio de los medicamentos requeridos. Por eso, las empresas de seguros (que no parecen ver la salud como un derecho sino como un mero bien de consumo) no están dispuestas a asumir tales pérdidas.

Estas son cosas que normalmente el público no cuestiona, no solo porque está acostumbrado a que estas entidades vendan contratos no negociables, sino también por lo engorroso que pueden ser los procedimientos administrativos o judiciales para cuestionar o impugnar algo así (resultaría caro en tiempo y dinero, apelar los rechazos de cobertura del seguro, ya sea ante la propia empresa 0 ante tribunales). Además, no se considera así quizás porque, en una sociedad que históricamente ha excluido y confinado la enfermedad mental, nadie quiere ser estigmatizado como si la sufriera, lo que dificulta que alguien desee exponerse al cuestionar de manera pública a una aseguradora, por esta arbitrariedad.

Por lo tanto, las aseguradoras deben modificar su postura de continuar excluyendo servicios básicos y, en el desafortunado caso de que no lo hagan, el Estado tiene la obligación de velar por la efectiva garantía del derecho humano a la salud en este ámbito particular. Se trata de un principio de fortalecimiento democrático, que de no alcanzarse, estaría propiciando que mujeres y hombres de todas las edades vean gravemente afectado su acceso a los diversos servicios de prevención y tratamiento en salud mental, y por ende, el efectivo e integral goce del derecho a la salud general.

\section{Referencias}

1. Organización Mundial de la Salud. (1992). Clasificación estadística internacional de enfermedades y problemas relacionados con la salud. 10a. revisión. Washington, D.C.: OMS

2. American Psychiatric Association. Diagnostic and Statistical Manual of Mental Disorders, 5th ed. Washington DC; American Psychiatric Association, 2013.

3. Mathers CD, Loncar D. Projections of global mortality and burden of disease from 2002 to 2030. PLoS Med 2006, 3: e442. doi: 10.1371/journal. pmed.00304424

4. Prince M, Patel V, Saxena S, Maj M, Maselko J, Phillips MR, et al. No health without mental health. Lancet 2007; 370 (9590): 859-77.

5. Diputados a la Asamblea Nacional Constituyente. Constitución Política de la República de Costa Rica. Artículo 46.7 de noviembre de 1949. También accesible en: http://pdba.georgetown.edu/Parties/CostaRica/Leyes/ constitucion.pdf. Consultado el 8 de marzo de 2015.

6. Protocolo a la Convención Americana sobre Derechos Humanos en Materia de Derechos Económicos, Sociales y Culturales "Protocolo de San Salvador". También accesible en: http://www.oas.org/juridico/spanish/tratados/a-52. html. Consultado el 8 de marzo de 2015. 